\title{
Prevalence of tinea corporis and tinea cruris in Chitradurga rural population
}

\author{
Girish V. Nagaral ${ }^{1, *}$, Veerabhadra Goud G.K ${ }^{2}$, Sudha .P3, Jagadevi ${ }^{4}$ \\ ${ }^{1,2}$ Associate Professor, ${ }^{1}$ Dept. of Dermatology, ${ }^{2}$ Dept of Biochemistry, Akash Institute of Medical Sciences \& Research Centre, \\ Devanahalli, Bangalore, ${ }^{3}$ Professor, Dept. of OBG, Akash Institute of Medical Sciences \& Research Centre, Devanahalli, \\ Bangalore, Karnataka, ${ }^{4}$ Assistant Professor, Dept. of Microbiology, Basaveshwara Medical College and Hospital, Chitradurga, \\ Karnataka, India
}

*Corresponding Author:

Email: derm288@gmail.com

\begin{abstract}
Introduction: Globally, the prevalence of superficial fungal skin infection is 20-25\%. Dermatophytes are the most common agents. In general population, tinea corporis and tinea cruris infections are very common. The present study is to find the prevalence of tinea corporis and tinea cruris in Chitradurga rural population.

Materials and Methods: This is a prospective observational study, conducted at Dermatology Department of Dermatology, Basaveshwara Medical College and Hospital, Chitradurga. A total of 200 patients showing lesions typical of dermatophytes infection were included in this study. Among 200 patients, 107 patients had tinea corporis, 50 patients had tinea cruris, 7 patients had tinea facia and 36 patients had other fungal infections. A detailed clinical history was taken from study subjects.

Result: In the present study, 200 patients were included in the study. Among them 107 patients were diagnosed as tinea corporis $(53.5 \%)$ and 50 patients had tinea cruris (25\%) infection. Out of 107 tinea corporis patients $76(71 \%)$ were males and $31(29 \%)$ were females. Out of 50 tinea cruris patients $35(70 \%)$ were males and $15(30 \%)$ were females. Tinea facia is diagnosed in 7 patients and 36 patients had other fungal infections. Tinea corporis is more prevalent in males than females.

Conclusion: Dermatophytosis, a common condition, especially tinea corporis and tinea cruris is public health problem affecting all age groups in our area. It is found that the prevalence of tinea corporis (53.5\%) is more in this population and the prevalence of tinea corporis is high in males (71\%) and in 21-30 years of age group. Use of non-occlusive absorbent cotton garments and good hygiene prevent this ring worm infection.
\end{abstract}

Keywords: Dermatophytes, Fungal Infections, Prevalence, Superficial fungal infections.

\section{Introduction}

Globally, the prevalence of superficial fungal skin infection is $20-25 \%$, dermatophytes are the most common agents to cause fungal infections and it is quite common. ${ }^{1,2}$ The prevalence of this infection is very high in the tropical countries. ${ }^{3,4}$ This fungal infection is prevalent in all age groups of both sexes in our country. ${ }^{5}$

The Dermatophytes are a group of closely related fungi that invade the keratinized tissue (skin, hair, and nail) of humans and other animals to cause an infection, known as dermatophytosis, referred to as "Ringworm" due to the appearance of the lesion or "Tinea". These species are of three types geophilic, zoophilic, or anthropophilic based on whether they predominantly reside in the soil, on animals, or on humans, respectively. ${ }^{3}$ Infection caused by dermatophytes is generally cutaneous and restricted to the nonliving cornified layers because the fungi is not able to penetrate the deeper tissues or organs of immunocompetent hosts. The infection spreads very easily by direct contact from infected humans and animals or through fomites. ${ }^{5}$

In humans, Dermatophytes manifest in different forms. Trichophyton species are the common agents to cause Tinea corporis and Tinea cruris infections. Trichophyton species disrupts keratin in the stratum corneum (epidermis of skin). This is because the dermatophyte group of fungi are not able to penetrate tissues which are not fully keratinized. However, the severity of dermatophyte infection varies from mild to moderate according to host immunity, virulence of the infecting species, site of infection and environmental factors. ${ }^{6}$

Worldwide, the incidence and distribution of these fungal infections varies significantly as the prevalence of different species varies with geographic regions, local cultural practices, climate and socioeconomic conditions. Overcrowding, areas with high humidity, poor hygienic conditions are the main factors, which predispose dermatophytosis. ${ }^{7}$

Dermatophyte infection of the glabrous skin of the trunk, shoulders and limbs (except scalp, groin, soles and palms) termed as tinea corporis and can be caused by Trichophyton species. It is the most common dermatophyte infection in India and abroad. ${ }^{4,6}$

In world, Trichophyton rubrum is the quite common infectious agent. $47 \%$ cases of Tinea corporis are caused by Trichophyton rubrum. It starts as a flat, scaly and more often as a pruritic macules which may further develop into a lesion with raised border spreading radially with erythematous vesicular edges. This may expand as a ring with central clearance and irregular circles giving this infection a layman name "ring worm". Tinea corporis occurs globally, and is relatively frequent, but its higher incidences are seen in tropics and subtropics. In India, tinea corporis is accountable for up to $88 \%$ of cases. Infection can occur 
from direct or indirect contact with skin and scalp lesions of infected persons or animals. ${ }^{6}$

Tinea cruris, another variety of superficial fungal infection, caused by Trichophyton rubrum, Epidermophyton floccosum which manifest as an inflammatory lesion or asymptomatic,(depends on species virulence and patient's immune response) most commonly in the groin and genital area (perianal and perineal sites) with a tendency to use keratin as its nitrogen source for growth. Lesions are erythematous and covered with thin, dry scales, can extend to inner thigh with raised, defined border with small vesicles. ${ }^{8,9}$

The diagnosis of tinea corporis is mainly clinical findings and occasionally laboratory investigations are also required such as direct microscopy of the specimen in $10 \% \mathrm{KOH}$ solution and fungal culture in Sabouraud's dextrose agar (SDS) medium. In Indian, there are various reports regarding the prevalence of the Dermatophyte infection. Although the dermatophyte infection is not invasive and easy to cure, its widespread nature and cost of the treatment is a major public health problem and burdens the economic status of the tropical countries like India. Few studies reported on the prevalence of the dermatophyte infections in Karnataka, India. Hence, the present study is to find the prevalence of tinea corporis and tinea cruris among different age groups in Chitradurga rural population.

\section{Materials and Methods}

This study is a prospective observational study, conducted at Outpatient Department of Dermatology, Venoreology and Leprology, Basaveshwara Medical College and Hospital, Chitradurga. After obtaining the Institutional Ethical Clearance and informed consent, a total number of 200 subjects were included in the study. Patients with complaint of itching or lesion were screened. Tinea corporis and tinea cruris were diagnosed clinically in terms of patient's history and clinical examination. ${ }^{10}$
A detailed clinical history was taken and physical examination done in all the study subjects. It included age, sex, site of infection, duration of disease, history of similar illness in family, socioeconomic status, occupation, history of recurrence, lifestyle and history of associated diseases were elicited and recorded in proforma. Patients those who had undertaken any topical or systemic antifungal treatment and nondermatophyte infections in the past 2 months at the time of presentation were excluded from the study.

To avoid a misdiagnosis, identification of dermatophyte infections from skin scraping is suggestible. Hence, the patient was taken to mycological unit of Microbiology department. Microscopic examination, consisting of a $10 \%$ to $15 \%$ $\mathrm{KOH}$ preparation, from skin scrapings from peripheral margins and roof of the blister using small scalpel blade, dermatophytes are easily recognized under microscope by their long branch like tubular structures called HYPHAE and it was taken as confirmatory test.

\section{Results}

In this study, Out of 200 patients, 107 patients had tinea corporis $(53.5 \%)$ infection and 50 had tinea cruris $(25 \%)$ infection. The initial diagnosis is done by clinically and after that mycological confirmation of presence of hyphae in $\mathrm{KOH}$ mounts under microscopy. Out of 107 tinea corporis patients 76 were males $(71 \%)$ and 31 were females (29\%) and 90 patients were $\mathrm{KOH}$ positive whereas 17 patients were $\mathrm{KOH}$ negative. The prevalence of tinea corporis is more in males compared to females. Out of 50 tinea cruris $(25 \%)$ patients, 35 were males $(70 \%)$ and 15 were females $(30 \%)$ and 35 patients were $\mathrm{KOH}$ positive whereas 15 patients were $\mathrm{KOH}$ negative. Tinea Facia is observed in 7 patients and other fungal infections were observed in 36 patients. (Table 1, Fig. 1 and 2)

Table 1: Details of patients with reference to sex and clinical manifestations

\begin{tabular}{|l|c|c|c|}
\hline \multirow{2}{*}{ Clinical Manifestation } & Total number of subjects & \multicolumn{2}{|c|}{ Sex } \\
\cline { 3 - 4 }$(\mathbf{n = 2 0 0})$ & Male & Female \\
\hline Tinea Corporis & $107(53.5 \%)$ & $76(71 \%)$ & $31(29 \%)$ \\
\hline Tinea Cruris & $50(25 \%)$ & $35(70 \%)$ & $15(30 \%)$ \\
\hline Tinea Facia & $7(3.5 \%)$ & $5(71.4 \%)$ & $2(28.5 \%)$ \\
\hline Other fungal infections & $36(18 \%)$ & $21(58.3 \%)$ & $15(41.6 \%)$ \\
\hline
\end{tabular}




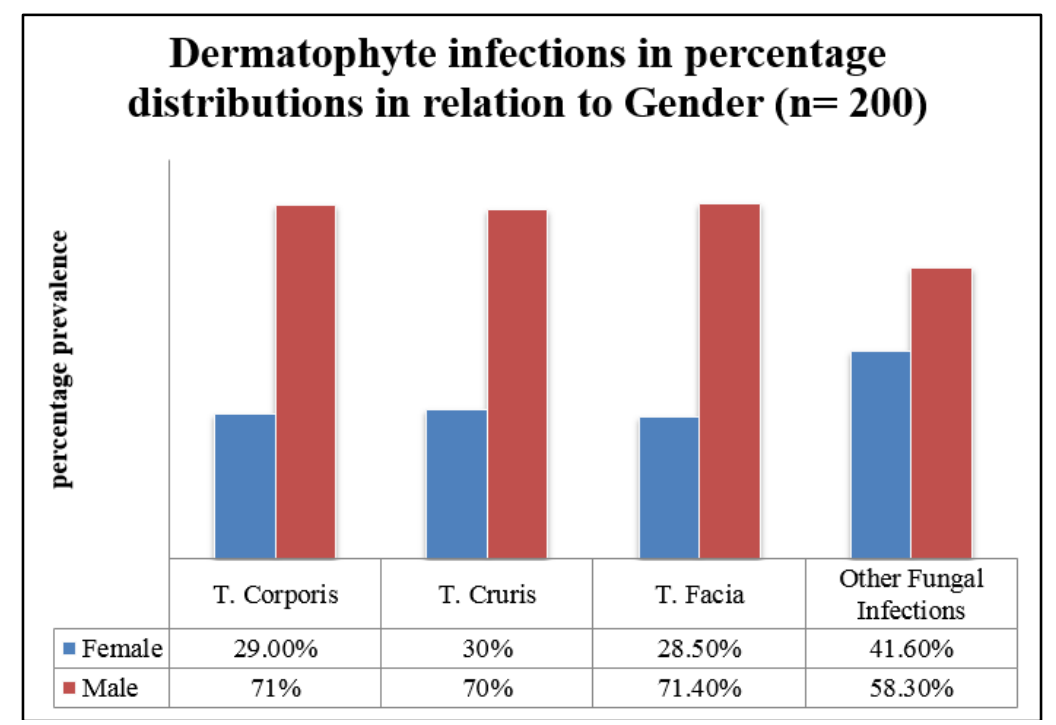

Fig. 1: Gender wise percentage distribution of dermatophyte

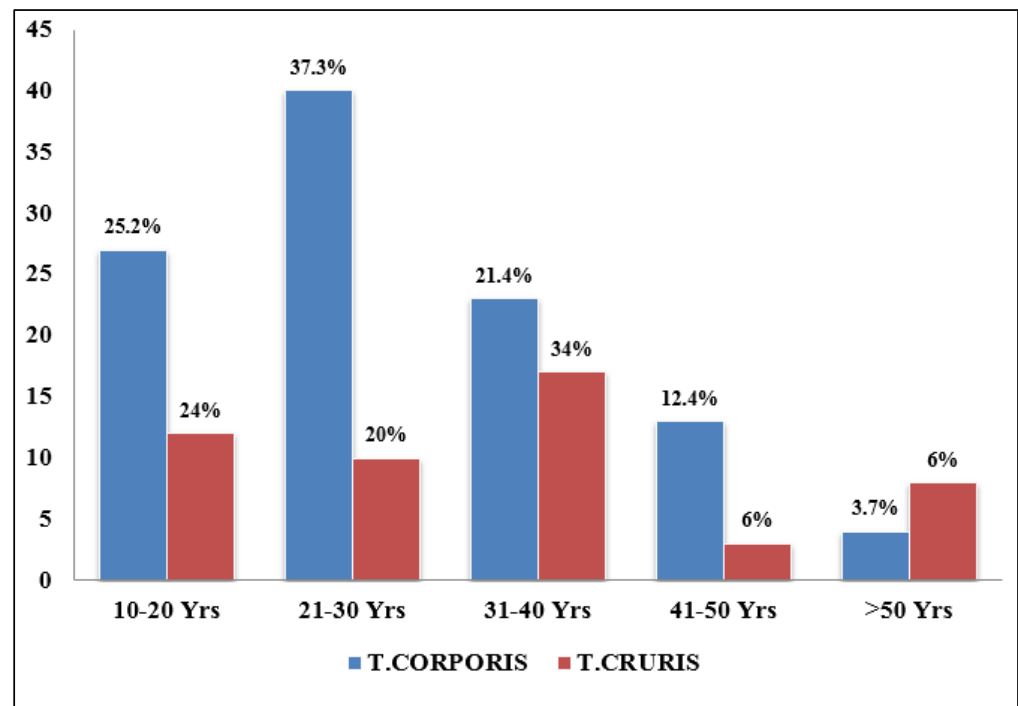

Fig. 2: Age wise distribution of T. corporis and T. cruris

\section{Discussion}

Studies have shown that high occurrence of dermatophytoses from different parts of the world. ${ }^{11}$ Globally, Dermatophyte infections are very common. Epidemiological features vary according to the geographical area as a consequence of migratory streams, lifestyle and socioeconomic conditions, and incidence of peculiar comorbidities. ${ }^{12}$ In recent decades, studies showed a growing etiological role of some anthropophilic dermatophytes has become evident all over the world. All races are usually affected and the clinical varieties and prevalence appear to depend mainly on environmental factors. ${ }^{13}$

India is a tropical country and it's climate is conducive for dermatophytosis. Finding the prevalence of Dermatophyte infections and their various clinical presentations helps in the early diagnosis and proper treatment of Dermatophytosis. ${ }^{14}$
In this study, 200 clinically diagnosed dermatophytoses cases were studied. Studies have reported that males are more frequently affected by dermatophytes infection compared to females ${ }^{5}$. In the present study, out of 200 patients, $137(68.5 \%)$ were males, which is higher than the females, $63(31.5 \%)$. The prevalence of $\mathrm{T}$. corporis and $\mathrm{T}$. cruris is more in males, $71 \%$ and $70 \%$ respectively. In males, the increased percentage may be due to increased outdoor exposure and more physical work, results in increased sweating and less cosmotic consciousness compared to females. ${ }^{3}$ In females, the lower incidence could also be due to non-reporting of females. Out of 200 patients, the highest prevalent dermatophyte infection was $\mathrm{T}$. corporis $(53.5 \%)$, followed by $\mathrm{T}$. cruris $(25 \%), \mathrm{T}$. capitis (3.5\%) and other fungal infections (18\%).

The study results showed that the prevalence of $\mathrm{T}$. corporis infection is high in the adult age group (21 - 30 years) and T. cruris in the age group of 31-40. The highest prevalence of this dermatophyte infection in 
this age group may be due to increased physical activity and this leads to excessive sweating which is more favorable for the dermatophytes growth. This study finding are supported by earlier studies. ${ }^{15-18}$

The present study results are comparable with the earlier study reports from Tamil Nadu, Madhya Pradesh, Manipal and Kashmir. ${ }^{19-21}$ Apart from India, in Brazil the prevalence of tinea corporis is reported to be most predominant clinical type. ${ }^{22-23}$ In men, tinea cruris is more prevalent compared to women. This findings were supported by earlier studies. ${ }^{24}$

The main reason behind the high prevalence of above mentioned groups i.e., tinea corporis and tinea cruris is the severe itching which induces the patient to seek medical advice. ${ }^{13}$ This is in agreement with majority of all cases of recurrent disease and extensive disease. Our study results are in accordance with studies done by. ${ }^{25-28}$ Dermatophytosis is mainly due to less aeration due to tight clothing, increased humidity and temperature of the body which makes skin as a suitable growth environment for dermatophytes, maceration, and sweating in groin and waist region. ${ }^{28}$ These conditions are shown to be associated with the incidence of tinea corporis and tinea cruris. ${ }^{26,18}$ The main limitations of our study is culture and sensitivity assays and details about other fungal infections. Further studies are recommended to find out the prevalence of other fungal infections in this population.

\section{Conclusion}

From the present study, it may be concluded that in tropical countries like India, Dermatophytosis is a common fungal infection, affecting all age groups in our area. This study, found that the tinea corporis is more prevalent than tinea cruris. Prevalence of tinea corporis is more in males and in 21-30 years of age group. Use of non-occlusive absorbent cotton garments and good hygiene prevent this ring worm infection. Patients need to be educated regarding the continuation of medications until mycological cure in order to prevent recurrence and also about the hygienic measures.

\section{Acknowledgements: Nil}

\section{Conflict of Interest: Nil}

\section{References}

1. Gebreabiezgi Teklebirhan and Adane Bitew. Prevalence of Dermatophytic Infection and the Spectrum of Dermatophytes in Patients Attending a Tertiary Hospital in Addis Ababa, Ethiopia. Hindawi Publishing Corporation. International Journal of Microbiology. 2015;2015:1-5.

2. Alok Kumar Sahoo, Rahul Mahajan. Management of tinea corporis, tinea cruris, and tinea pedis: A comprehensive review. Indian Dermatology Online Journal. 2016;7:77-86.

3. Vijayakumar Ramaraj, Rajyoganandh S Vijayaraman, Sudha Rangarajan, Anupma Jyoti Kindo. Incidence and prevalence of dermatophytosis in and around Chennai, Tamilnadu, India. International Journal of Research in Medical Sciences. 2016;4(3):695-700.

4. Muhilan Jegadeesan, Sheela Kuruvila, Shashikala Nair. Clinico-etiological Study of Tinea Corporis: Emergence of Trichophyton mentagrophytes. International Journal of Scientific Study. 2017;5(1):161-165.

5. Uma Penmetcha, Ramesh Babu Myneni, Padmaja Yarlagadda and Susmitha Simgamsetty. A Study of Prevalence of Dermatophytosis in and around Guntur District, Andhra Pradesh, South India. International Journal of Current Microbiology and Applied Sciences. 2016;5(9):702-717.

6. Brigida S and Muthiah NS. Prevalence of Tinea Corporis and Tinea Cruris in Outpatient Department of Dermatology Unit of a Tertiary Care Hospital. Journal of Pharmacology and Clinical Research. 2017;3(3):001003.

7. Ranganathan S, Menon T, Selvi SG, Kamalam A. Effect of socio-economic status on the prevalence of dermatophytosis in Madras. Indian Journal of Dermatology, Venereology and Leprology. 1995;61:1618.

8. Ameen M. Epidemiology of superficial fungal infections. Clinical Dermatology. 2010; 28: 197-201.

9. Asticcioli S, Di Silverio A, Sacco L, Fusi I, Vincentil, Romero E. Dermatophyte infections in patients attending a tertiary care hospital in northern Italy. New Microbiol. 2008;31(4):543-548.

10. Gupta A. K., Bluhm R., Summerbell R., Pityriasis versicolor. Journal of European Academy of Dermatology and Venereology. 2000; 16: 19-33.

11. Anup Kainthola, Puneet Gaur, Alkesh Dobhal and Shailendra Sundriyal. Prevalence of Dermatophytoses in Rural Population of Garhwal Himalayan Region, Uttarakhand, India. International Research Journal of Medical Sciences. 2014;2(8):9-12.

12. Gino A. Vena, Paolo Chieco, Filomena Posa, Annarita Garofalo, Anna Bosco, Nicoletta Cassano. Epidemiology of dermatophytoses: retrospective analysis from 2005 to 2010 and comparison with previous data from 1975. New Microbiologica. 2012;35,207-213.

13. Seema Bose, Rekha Barapatre, Atindra Krishna Ghosh. Clinico - mycological profiles of dermatophytoses in a tertiary care rural hospital. International Journal of Biomedical and Advance Research. 2013;4(01):31-34.

14. Sudha M, Ramani CP, Heber Anandan. Prevalence of Dermatophytosis in Patients in A Tertiary Care Centre. International Journal of Contemporary Medical Research. 2016;3(8):2399-2401.

15. Kumar K, Kindo AJ, Kalyani J, Anandan S. ClinicoMycological Profile of Dermatophytic Skin Infections In A Tertiary Care Center-A Cross Sectional Study. Sri Ramachandra Journal of Medicine. 2007;1(2);12-15.

16. Verenkar MP, Pinto MJW, Rodrigues S, Roque WP, Singh I. Clinico-Microbiological study of dermatophytoses. Indian Journal of Pathology and Microbiology. 1991;34(3):186-192.

17. Senthamilselvi G, Kamalam A, Thambiah AS. Scenario of chronic dermatophytosis. Mycopathologia. 1998;140:129-135.

18. Singh, S. and Beena, P.M.: Profile of dermatophyte infections in Baroda. Indian Journal of. Dermatology, Venerology and Leprology. 2003;69(4):281-283.

19. Kumar K, Kindo AJ, Kalyani J, Anandan S. ClinicoMycological Profile of Dermatophytic Skin Infections In A Tertiary Care Center-A Cross Sectional Study. Sri Ramachandra Journal of Medicine. 2007;1(2);12-15. 
20. Venkatesan G, Ranjit Singh AJA, Murugesan AG, Janaki C, Gokul Shankar S. Trichophyton rubrum-the predominant etiological agent in human dermatophytoses in Chennai, India. African Journal of Microbiology Research. 2007;1(1);9-12.

21. Balakumar, Srinivasan. Epidemiology of dermatophytosis in and around Tiruchirapalli, Tamilnadu, India. Asian Pacific Journal of Tropical Diseases. 2012;2(4):286-289.

22. Chinelli PA, Sofiatti Ade A, Nunes RS, Martins JE. Dermatophyte agents in the city of Sao Paulo, from 1992 to 2002. Revista do Instituto do Medicina Tropical do Sao Paulo. 2003;45:259-263.

23. Fortuno B, Torres L, Simil E, Seoane A, Uriel JA, Santacruz C. Dermatophytes isolated in our clinics, 5year study in Zaragoza. Enfermededes Infecciosas Y Microbiologia Clinica. 1997; 15:536-539.

24. Garg A, Venkatesh V, Singh M, Pathak KP, Kaushal GP, Agrawal SK. Onychomycosis in central India: a clinicoetiologic correlation. Int J Dermatol. 2004;43:498502 .

25. Bindu, V. and Pavithran, K. Clinico - mycological study of dermatophytosis in Calicut. Indian Journal of
Dermatology, Venerology and Leprology. 2002;68(5):259-261.2.

26. .Ranganathan, S., Menon, T. and Sentamil, G.S. Effect of socio-economic status on the prevalence of dermatophytosis in Madras. Indian Journal of. Dermatology, Venerology and Leprology. 1995;61(1):1618.

27. Seebacher C, Bouchara JP, Mignon B. Updates on the epidemiology of dermatophyte infections. Mycopathologia. 2008;166:335-352.

28. Kamothi, M. N., Prevalence of Dermatophyte Infection in District Rajkot, Electronic. Journal of Pharmacology and Therapy. 2010;3:1-3.

How to cite this article: Nagaral GV, Veerabhadra GGK, Sudha .P, Jagadevi. Prevalence of tinea corporis and tinea cruris in Chitradurga rural population. Ind $\mathrm{J}$ Clin Exp Dermatol. 2018;4(3):221-225. 